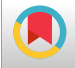

\title{
Real World Experience with All-Oral Interferon Free Regimen for the Treatment of Lebanese Patients with Hepatitis C Virus (HCV) Infection Antoine Abou Rached ${ }^{1,2,{ }^{*}}$, Jowana Saba ${ }^{1}$, Cesar Yaghi ${ }^{3}$, Ala Sharara ${ }^{4}$ and Walid Ammar ${ }^{2}$ \\ ${ }^{1}$ Lebanese University, School of Medicine, Beirut, Lebanon \\ ${ }^{2}$ Ministry of Public Health, Beirut, Lebanon \\ ${ }^{3}$ Saint Joseph University, School of Medicine, Beirut, Lebanon \\ ${ }^{4}$ American University of Beirut, School of Medicine, Beirut, Lebanon \\ "Corresponding author: Antoine Abou Rached, Lebanese University, School of Medicine, Beirut, Lebanon. Tel: +96-13746317, E-mail: abourachedantoine@gmail.com
}

Received 2018 April 21; Revised 2018 June 11; Accepted 2018 June 16.

\begin{abstract}
Objectives: The treatment of hepatitis $\mathrm{C}$ has dramatically improved since the introduction of new direct-acting antivirals (DAAs). The aim of this study was to assess the efficacy and safety of all oral DAAs, with or without ribavirin, in the treatment of naive and treatment experienced hepatitis C virus (HCV) Lebanese patients.

Methods: This study reviewed all cases approved for hepatitis C treatment with DAAs, according to Lebanese guidelines for treatment of HCV at the Ministry of Public Health from October 2015 to December 2016. Available data on age, gender, genotype (GT) and subtype, fibrosis stage, previous treatment (if present), new DAAs treatment, and sustained virological response at week 12 (SVR12) were collected.

Results: During a period of 15 months, a total of 186 patients were treated with DAAs. In total, 57\% were male. The mean age of the patients was 54.3 years. Genotype 1 was the most prevalent (45\%), followed by genotype four (34\%) and genotype three (12\%). More than $72 \%$ of patients had advanced fibrosis (F3-F4) before starting DAAs and $42 \%$ of patients were treatment experienced. Regarding the different DAAs protocols used, SVR12 was achieved in 93\% of cases, while $4 \%$ did not achieve SVR. Furthermore, $3 \%$ of cases were either lost to follow up or had major adverse events. Sustained virological response at week 12 was $93 \%, 96 \%$, and 94\% in GT1, GT3, and GT4, respectively. In cirrhotic patients, SVR12 was 90\%. There was no difference in SVR12 between treatment naive and treatmentexperienced patients. Hepatocellular carcinoma developed in five patients during the period of the study.

Conclusions: This is the first real world Lebanese data concerning hepatitis C treatment with DAAs. It showed a satisfactory response rate irrespective of previous treatments or the stage of fibrosis.
\end{abstract}

Keywords: Hepatitis C, Fibrosis, Genotype, Antivirals

\section{Background}

Hepatitis C virus (HCV) is considered as the most common cause of liver disease in the world, and HCV-related complications account for approximately 700,000 deaths each year (1). Chronic HCV infection is a major cause of death and morbidity due to serious liver disease complications, including cirrhosis and hepatocellular carcinoma (HCC) (2). Indeed, recent data suggests that the global burden of viral hepatitis has now surpassed many other common infectious diseases, such as tuberculosis, Acquired Immunodeficiency Syndrome (AIDS), diarrheal disease, and malaria (3).

Successful eradication of HCV reduces liver-related morbidity and mortality (4). Achieving a sustained virological response (SVR) with antiviral therapy, defined as undetected HCV RNA measured at week 12 or 24 post-treatment, is associated with a lower risk of developing HCC (4), and a lower all-cause mortality in patients with advanced fibro- $\operatorname{sis}(5)$.

The interferon (INF) free direct-acting antivirals (DAAs) are currently the standard management of hepatitis $C$. These medications have an excellent tolerability, while achieving a high SVR.

Real-world evidence is important to study the degree of concordance between clinical trial data and common daily practice results. It may provide guidance in clinical decision-making in a particular setting.

\section{Objectives}

In this cohort, the researchers studied the efficacy of DAAs in Lebanese patients infected with different HCV genotype (GT), who were treated according to the Lebanese guidelines (6). 


\section{Methods}

In this retrospective cohort study, the researchers reviewed charts of all patients chronically infected with HCV, who were treated at the ministry of public health (MOPH) with a DAA-containing therapy, between July 2015 and December 2016. Patient demographics (age and gender), detailed HCV virologic characteristics (GT and subtype), fibrosis stage determined by non-invasive tests (elastography) and/or liver biopsy, presence of comorbidities, and prior HCV treatment experience were recorded. All Lebanese patients between 18 and 80 years old with chronic HCV infection were included in the study. Non-Lebanese patients and/or those aged more than 80 years were excluded from the study. Treatment decisions were based on the presence of comorbidities and the severity of liver disease as estimated by the fibrosis stage. The planned duration of therapy ranged from 12 to 24 weeks, with or without the use of Ribavirin (RBV), according to the HCV genotype and the patients characteristics (cirrhotic, naive or treatment experienced, etc.). All treatment regimens were selected based on the Lebanese guidelines for the management of HCV. The SVR at 12 weeks was used as the primary end point in this study by using the COBAS TAQMAN HCV monitor test (Roche diagnostics). The aim was to assess the efficacy of the treatment with DAAs in naive and treatment experienced patients.

The MOPH approved this retrospective study, without written informed consent from the patients, as the collected data was anonymous.

Descriptive statistics were presented, given the retrospective database design of the study. Descriptive statistics were generated using as numbers and percentages for categorical variables and means (standard deviation [SD]) for continuous variables. Fisher's 95\% confidence intervals were calculated for SVR rates. Multivariate logistic regression modeling included a priori compiled list of variables (age, gender, and genotype). All statistical analyses were performed using IBM SPSS Statistics version 22.

\section{Results}

A total of 186 patients were enrolled; $43 \%$ were females and 57\% males. The mean age was 54.3 years (range: 22 to 80 ). The most common GT was GT1 in $44.6 \%$ (subtypes 1a: $27.7 \%$ and 1 b: $57 \%$, untypable: $15.3 \%$ ), while GT2, GT3, GT4, GT5, and GT6 were identified in $6.45 \%, 12.4 \%, 34.4 \%, 1.6 \%$, and $0.5 \%$; respectively. Fibrosis stages distribution was as follows: F0: 8.1\%, F1:5.9\%, F2:12.4\%, F3: 15.1\%, F4/cirrhosis: 56.5\%, and unknown in $2.4 \%$.

Only $58.1 \%$ of patients were treatment naive whereas $41.9 \%$ of patients were treatment experienced (with pegy- lated INF and RBV in $88.5 \%$, DAAs first generation in $6.4 \%$ and DAAs second generation in 5.1\%). Treatment regimen used per genotype is presented in Table 1 . Overall, RBV was used in 51.6\% of cases. Treatment was completed in $96.7 \%$. It was discontinued due to major side effects (acute renal failure, long term fever, and hepatic encephalopathy) in $1.6 \%$. Three patients were lost to follow up (1.6\%).

The SVR 12 was achieved in 93\% of cases (173 out of 186 patients). If those lost to follow up were excluded, SVR12 would increase to 94\%. Polymerase Chain Reaction (PCR) HCV remained positive at week 12 post-treatment in $3.8 \%$ of patients ( 7 out of 186 patients). All these patients were cirrhotic except one, who took a wrong dose of medication. In multivariate logistic regression analysis, neither age, gender, race, nor genotype reveal significant impact of SVR 12. In total, five out of 186 patients (2.9\%) developed HCC after receiving the treatment and during this study. The results are shown in Table 2.

After exclusion of patients lost to follow up, SVR12 rates were $94 \%, 100 \%, 95.7 \%, 93.8 \%, 100 \%$, and $100 \%$ in GT1, GT2, GT3, GT4, GT5 and GT6, respectively (Figure 1). According to fibrosis stage, SVR12 was $100 \%$ in Fo, F1, and F3, 95.65\% in F2, and $90.5 \%$ in F4 with no significant difference $(P=0.839)$. The SVR12 rate in the three most common genotypes and per fibrosis stage is represented in Figure 2. There was no significant difference in SVR12 between all GT in treatment naive and treatment-experienced patients (SVR12 was $94.4 \%$ and $93.6 \%$, respectively, with a $\mathrm{P}$ value of 0.97). No difference in SVR 12 was noted between all used DAAs regimens: Sofosbuvir/Ledipasvir (SOF/LED) +/- RBV: 95.8\% (GT1: 92.9\%, GT4: 100\%, GT5: 100\%), Sofosbuvir/Daclatasvir (SOF/DCL) +/- RBV: 96.7\% (GT1: 100\%, GT2: 100\%, GT3: 95\%, GT4: 100\%, GT5: 100\%), Sofosbuvir/Velpatasvir (SOF/VEL): 100\%, Sofosbuvir (SOF) + RBV: 100\%, Ombitasvir /Paritaprevir/r (OBV/PTVr) + RBV: 93.3\% (GT4) and Ombitasvir/Paritaprevir/r (OBV/PTVr) + Dasabuvir (DSV) +/- RBV: 97\% (GT1).

Only three patients developed major adverse events (acute renal failure, hepatic encephalopathy, and long term fever) that led to treatment cessation.

\section{Discussion}

The prevalence of HCV in Lebanon is low (0.2\%) (7) with GT1 being the most prevalent genotype, followed by GT4 then GT3 (8). This study highlighted the demographics of the HCV epidemic in Lebanon. It also provided the realworld Lebanese experience in the treatment of HCV with the new DAAs regimen.

It has been established that real-world evidence can provide insight into the efficacy and safety of therapeutic regimens in a broader patient population and a more 


\begin{tabular}{|c|c|c|c|c|c|c|}
\hline & SOF + RBV & LDV/SOF +/- RBV & SOF/DCL $+/-$ RBV & SOF/VEL $+/-$ RBV & $\mathbf{O M V} / \mathbf{P T V} / \mathbf{r}+\mathbf{D S V}+\mid-\mathbf{R B V}$ & $\mathbf{O M V} / \mathbf{P T V} / \mathbf{r}+\mathbf{R B V}$ \\
\hline GT1 Untypable & 0 & $12(92)$ & $1(8)$ & 0 & 0 & 0 \\
\hline GT1A & 0 & $11(48)$ & $1(4)$ & $1(4)$ & $10(43)$ & 0 \\
\hline GT1B & 0 & $20(43)$ & $2(4)$ & 0 & $25(53)$ & 0 \\
\hline GT2 & $11(92)$ & 0 & $1(8)$ & 0 & 0 & 0 \\
\hline GT3 & $3(13)$ & 0 & $20(87)$ & 0 & 0 & 0 \\
\hline GT4 & 0 & $27(42)$ & $4(6)$ & $1(2)$ & 0 & $32(50)$ \\
\hline GT5 & 0 & $2(67)$ & $1(33)$ & 0 & 0 & 0 \\
\hline GT6 & 0 & 0 & 0 & $1(100)$ & 0 & 0 \\
\hline
\end{tabular}

Abbreviations: DCL, Daclatasvir; DSV, Dasabuvir; GT, Genotype; LDV, Ledipasvir; OMV, Ombitasvir; PTV, Paritaprevir; r, Ritonavir; RBV, Ribavirin; SOF, Sofosbuvir; VEL, Velpatasvir.

${ }^{\mathrm{a}}$ Values are expressed as No. (\%).

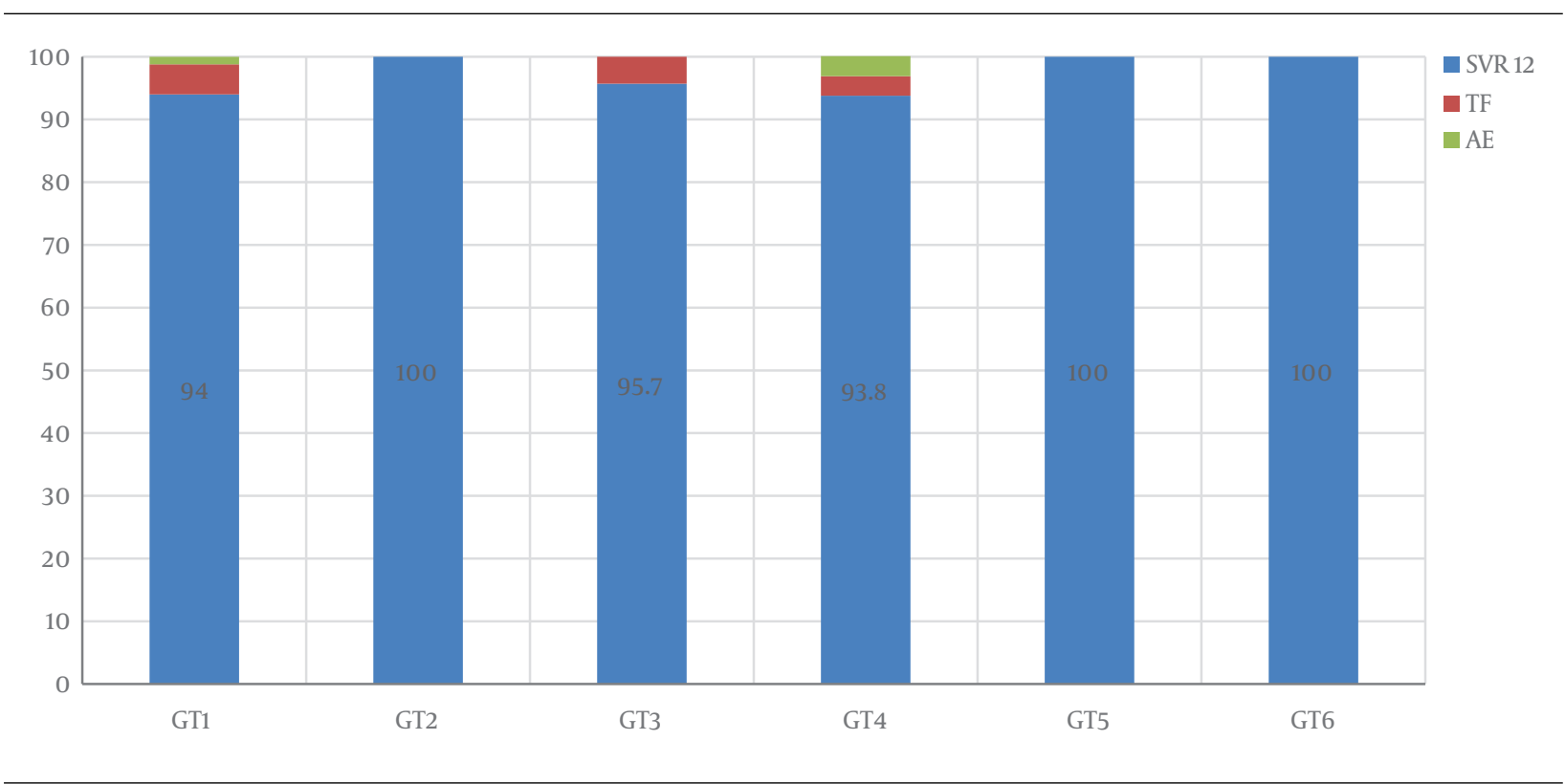

Figure 1. Sustained virologic response at week 12 per genotype. SVR12: sustained virologic response at 12 weeks post treatment; TF: treatment failure; AE: adverse events.

diverse clinical setting. Furthermore, DAAs have transformed therapy for HCV making cure possible for most patients. The current "real-world" national retrospective cohort study shows remarkable SVR rates of $94 \%$.

In this study, with a significant number of hard-totreat patients (liver cirrhosis and treatment experienced patients), the results were comparable to SVR rates of published data. Nevertheless, patients with liver cirrhosis showed a lower response rate (91\%), compared to patients without cirrhosis (98\%). Treatment-experienced patients and those infected with GT3 responded well to the new DAAs with cure rates of $89.8 \%$ and $95.5 \%$, respectively. There was no difference in the SVR rate between the different protocols of DAAs used. In GT1 and GT4 patients, there was no difference in SVR rates between the two regimens used: $\mathrm{OBV} / \mathrm{PTV} / \mathrm{r}+\mathrm{DSV}$ and SOF/VEL.

Comparably, a large cohort study, including 2099 patients, showed that $95 \%$ to $97 \%$ of cases infected with GT1 and those, who were treated with SOF/LDV \pm RBV for eight, twelve, or 24 weeks achieved SVR12 (9). Terrault et al. showed that SVR rates were more than $90 \%$ in treatment naive patients infected with GT1 HCV, who were treated with LDV/SOF-based regimen, and that there was no benefit from the use of RBV, which was associated with high rates of drug withdrawal (10). In Germany, in a large multicenter clinical practice cohort, including patients treated with the OBV/PTV/r \pm DSV \pm RBV and infected with GT1 or GT4, showed overall SVR12 rates ranging from $95 \%$ to 


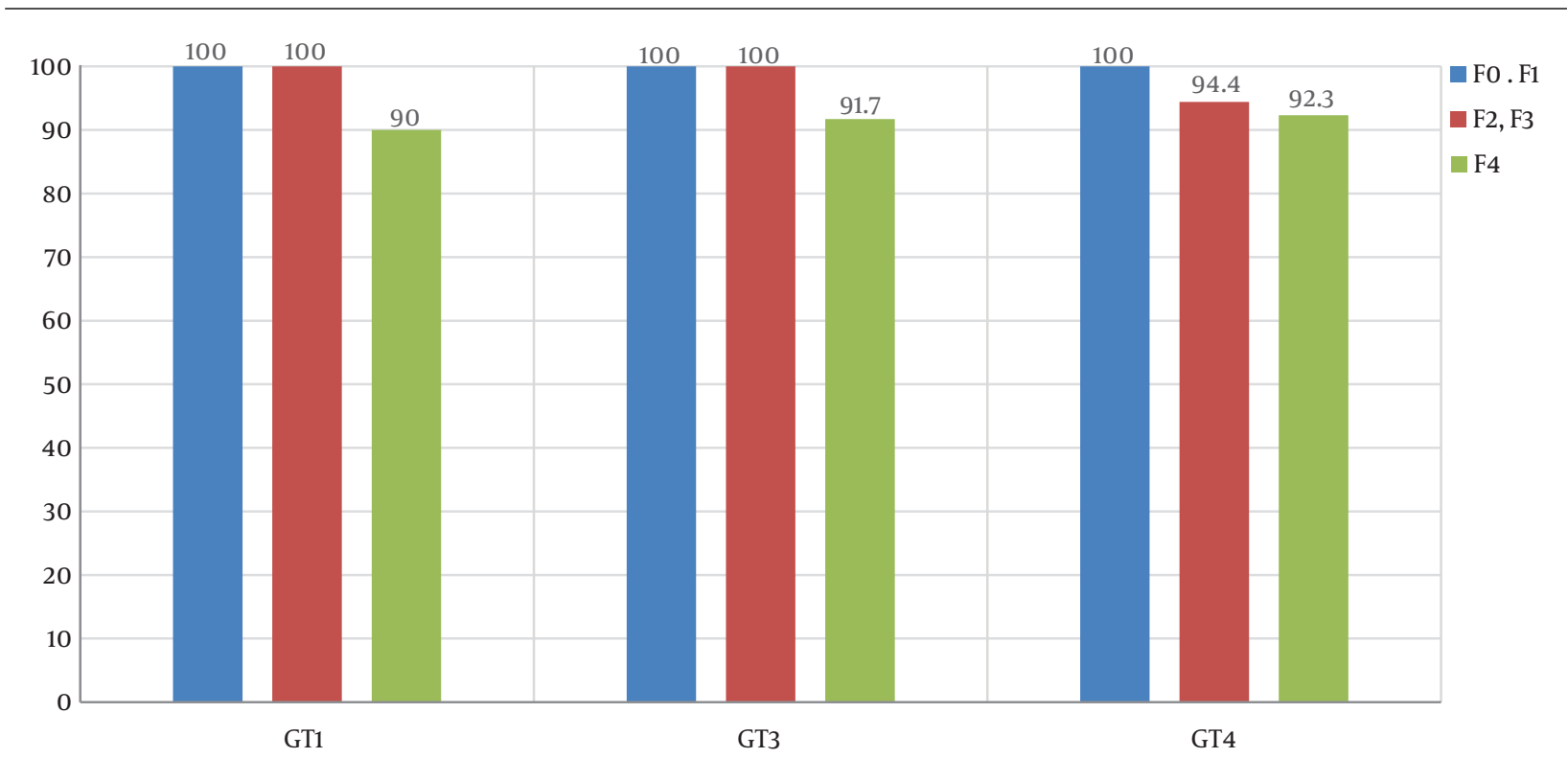

Figure 2. Sustained virologic response at week 12 per predominant genotype and fibrosis stage. F0 - F4: metavir fibrosis score Fo to F4; GT: genotype.

\begin{tabular}{|c|c|}
\hline Results & No. (\%) \\
\hline Patients who continued therapy & $180(96.7)$ \\
\hline Lost to follow up & $3(1.6)$ \\
\hline Treatment stopped because of adverse event & $3(1.6)$ \\
\hline SVR12 achieved & $173(93)$ \\
\hline HCV recurrence 12 weeks after treatment completion & $7(3.8)$ \\
\hline \multicolumn{2}{|l|}{2 GT1: F4 (1treatment experienced) } \\
\hline \multicolumn{2}{|l|}{2 GT1a: F4 (2 treatment experienced) } \\
\hline \multicolumn{2}{|l|}{ 1 GT3: F4 (naive) } \\
\hline \multicolumn{2}{|l|}{$2 \mathrm{GT} 4: 1 \mathrm{~F} 2,1 \mathrm{~F} 4$ (naive) } \\
\hline HCC & $5(2.9)$ \\
\hline SVR12 achieved & $4(2.6)$ \\
\hline PCR positive at week 12 & $1(0.3)$ \\
\hline
\end{tabular}

$100 \%$. The clinical efficacy did not markedly differ in cirrhotics or in previously treated patients. The SVR12 in nonresponders to interferon reached $98 \%$ (11). Another study provided real-world evidence for an excellent anti-viral potency of OBV/PTV/r \pm DSV \pm RBV in the treatment of $\mathrm{HCV}$ GT1 and 4 infection. Viral eradication success was achieved in $99 \%$ of patients, with SVR12 rates ranging from $96.4 \%$ to $100 \%$ between subgroups (12).
A meta-analysis of real-world data from over 5000 patients showed that OBV/PTV/r \pm DSV \pm RBV treatment resulted in SVR in $96.8 \%$ of patients with HCV GT1 or GT4 infection. Neither cirrhosis nor prior HCV treatment had statistically significant impact on SVR rates. The real-world relapse rate was around $1 \%$ in nine studies with 3,500 patients (13). The treatment was very effective in difficultto-treat populations, such as those with liver cirrhosis or null-responders to previous HCV treatment. Furthermore, SVR12 was achieved in 98.3\% (117 out of 119) of patients with liver cirrhosis irrespective of their treatment history (12).

In Spain, a large cohort study showed that HCV GT1infected patients treated with OMV/PTV/r + DSV or LDV/SOF had a high SVR12, despite the inclusion of a high proportion of patients with cirrhosis and prior treatment failure (14). In a study comparing the efficacy of LDV/SOF \pm RBV versus $\mathrm{OBV} / \mathrm{PTV} / \mathrm{r}+\mathrm{DSV} \pm \mathrm{RBV}$ in GT1 HCV-infected veterans, high SVR rates were achieved with both regimens ( $86 \%$ to $95 \%$ ) and within subgroups ( $83 \%$ to $100 \%$ ) (15). Another study showed no difference in SVR12 between the two regimens (OBV/PTV/r + DSV, SOF/VEL) in patients with HCV GT1 (16).

For GT4, Crespo et al. found that $96.2 \%$ of patients treated with $\mathrm{OMV} / \mathrm{PTV} / \mathrm{r} \pm \mathrm{RBV}$ and $95.4 \%$ treated with LDV/SOF \pm RBV achieved SVR12. Particularly in cirrhotic, SVR12 was $91.2 \%$ with OMV/PTV/r \pm RBV and $93.2 \%$ with $\mathrm{LDV} / \mathrm{SOF} \pm \mathrm{RBV}$. No significant difference was found in SVR12, according to the fibrosis stage (17).

In HCV GT3-infected patients, who received modern 
DAA-based treatment regimens in a real-world setting, alloral DAA-combinations, including two different DAAs displayed a significantly higher effectiveness compared to therapy regimens, which included only SOF in combination with RBV. High rates of SVR12 were achieved even in patients, who were generally considered "difficult-totreat", such as patients with cirrhosis or those previously treated, and in HIV co-infected patients. Moreover, SVR12 rates in the real world GECCO cohort were lower compared to phase III trials with comparable DAA regimes, yet were much higher than rates reported in the era of dual PEG/RBV-based therapy real world cohorts (18-20).

In conclusion, data from the current Lebanese cohort showed a satisfactory response rate irrespective of previous treatments or stage of fibrosis. The treatment of patients infected with HCV and previously excluded by the recommendations is currently being done. A major effort to identify all infected patients through screening the at risk population is essential for eradicating $\mathrm{HCV}$ infection in Lebanon.

\section{References}

1. WHO. Guidelines for the Screening Care and Treatment of Persons with Chronic Hepatitis CInfection: Updated Version. Geneva: WHO Guidelines Approved by the Guidelines Review Committee; 2016.

2. Westbrook RH, Dusheiko G. Natural history of hepatitis C. J Hepatol. 2014;61(1 Suppl):S58-68. doi: 10.1016/j.jhep.2014.07.012. [PubMed: 25443346].

3. Stanaway JD, Flaxman AD, Naghavi M, Fitzmaurice C, Vos T, Abubakar I, et al. The global burden of viral hepatitis from 1990 to 2013: findings from the Global Burden of Disease Study 2013. Lancet. 2016;388(10049):1081-8. doi: 10.1016/S0140-6736(16)30579-7. [PubMed: 27394647]. [PubMed Central: PMC5100695].

4. Hiramatsu N, Oze T, Takehara T. Suppression of hepatocellular carcinoma development in hepatitis $\mathrm{C}$ patients given interferon-based antiviral therapy. Hepatol Res. 2015;45(2):152-61. doi: 10.1111/hepr.12393. [PubMed: 25052449].

5. van der Meer AJ, Veldt BJ, Feld JJ, Wedemeyer H, Dufour JF, Lammert $\mathrm{F}$, et al. Association between sustained virological response and all-cause mortality among patients with chronic hepatitis C and advanced hepatic fibrosis. JAMA. 2012;308(24):2584-93. doi: 10.1001/jama.2012.144878. [PubMed: 23268517].

6. LSGE. The 14th annual congress of Lebanese society of gastroenterology. 2012. Available from: http://www.lsge.org/index.php?id=meetings\& name $=$ Meetings $\&$ type $=$ meetings.

7. Abou Rached A, Abou Kheir S, Saba J, Ammar W. Epidemiology of hepatitis B and hepatitis C in Lebanon. Arab J Gastroenterol. 2016;17(1):2933. doi:10.1016/j.ajg.2016.01.002. [PubMed: 27032492].

8. Abou Rached A, Yaghi C, Khalil L, Saba J, Ammar W. Prevalence of hepatitis $C$ virus genotypes and subtypes in Lebanese population and major high risk groups. Arab J Gastroenterol. 2017;18(2):114-7. doi: 10.1016/j.ajg.2017.05.001. [PubMed: 28579342].

9. Backus LI, Belperio PS, Shahoumian TA, Loomis TP, Mole LA. Realworld effectiveness of ledipasvir/sofosbuvir in 4,365 treatment-naive, genotype 1 hepatitis C-infected patients. Hepatology. 2016;64(2):40514. doi: 10.1002/hep.28625. [PubMed: 27115523].

10. Terrault NA, Zeuzem S, Di Bisceglie AM, Lim JK, Pockros PJ, Frazier LM, et al. Effectiveness of Ledipasvir-Sofosbuvir Combination in Patients With Hepatitis C Virus Infection and Factors Associated With Sustained Virologic Response. Gastroenterology. 2016;151(6):1131-1140 e5. doi: 10.1053/j.gastro.2016.08.004. [PubMed: 27565882]. [PubMed Central: PMC5300778].

11. Welzel TM, Hinrichsen H, Sarrazin C, Buggisch P, Baumgarten A, Christensen S, et al. Real-world experience with the all-oral, interferon-free regimen of ombitasvir/paritaprevir/ritonavir and dasabuvir for the treatment of chronic hepatitis $C$ virus infection in the German Hepatitis C Registry. J Viral Hepat. 2017;24(10):840-9. doi: 10.1111/jvh.12708. [PubMed: 28342229].

12. Flisiak R, Janczewska E, Wawrzynowicz-Syczewska M, Jaroszewicz J, Zarebska-Michaluk D, Nazzal K, et al. Real-world effectiveness and safety of ombitasvir/paritaprevir/ritonavir +/- dasabuvir +/- ribavirin in hepatitis C: AMBER study. Aliment Pharmacol Ther. 2016;44(9):94656. doi: 10.1111/apt.13790. [PubMed: 27611776].

13. Wedemeyer H, Craxi A, Zuckerman E, Dieterich D, Flisiak R, Roberts SK, et al. Real-world effectiveness of ombitasvir/paritaprevir/ritonavir+/dasabuvir+/-ribavirin in patients with hepatitis $C$ virus genotype 1 or 4 infection: A meta-analysis. J Viral Hepat. 2017;24(11):936-43. doi: 10.1111/jvh.12722. [PubMed: 28480525].

14. Calleja JL, Crespo J, Rincon D, Ruiz-Antoran B, Fernandez I, Perello C, et al. Effectiveness, safety and clinical outcomes of direct-acting antiviral therapy in HCV genotype 1 infection: Results from a Spanish real-world cohort. J Hepatol. 2017;66(6):1138-48. doi: 10.1016/j.jhep.2017.01.028. [PubMed: 28189751].

15. Backus LI, Belperio PS, Shahoumian TA, Loomis TP, Mole LA. Comparative effectiveness of ledipasvir/sofosbuvir +/- ribavirin vs. ombitasvir/paritaprevir/ritonavir + dasabuvir +/- ribavirin in 6961 genotype 1 patients treated in routine medical practice. Aliment Pharmacol Ther. 2016;44(4):400-10. doi: 10.1111/apt.13696. [PubMed: 27291852].

16. Walker DR, Pedrosa MC, Manthena SR, Patel N, Marx SE. Early View of the Effectiveness of New Direct-Acting Antiviral (DAA) Regimens in Patients with Hepatitis C Virus (HCV). Adv Ther. 2015;32(11):1117-27. doi: 10.1007/s12325-015-0258-5. [PubMed: 26538232]. [PubMed Central: PMC4662718].

17. Crespo J, Calleja JL, Fernandez I, Sacristan B, Ruiz-Antoran B, Ampuero J, et al. Real-World Effectiveness and Safety of Oral Combination Antiviral Therapy for Hepatitis C Virus Genotype 4 Infection. Clin Gastroenterol Hepatol. 2017;15(6):945-949 e1. doi: 10.1016/j.cgh.2017.02.020. [PubMed: 28238958].

18. Wehmeyer MH, Ingiliz P, Christensen S, Hueppe D, Lutz T, Simon $\mathrm{KG}$, et al. Real-world effectiveness of sofosbuvir-based treatment regimens for chronic hepatitis $C$ genotype 3 infection: Results from the multicenter German hepatitis C cohort (GECCO-03). J Med Virol. 2018;90(2):304-12. doi: 10.1002/jmv.24903. [PubMed: 28710853].

19. Schulze Zur Wiesch J, Pudelski N, Hoepner L, Supplieth M, Buggisch P, Lohse AW, et al. "Real-Life" comparison of pegylated-interferon 2a versus $2 \mathrm{~b}$ combination therapy of chronic hepatitis $\mathrm{C}$ virus. Hepatology. 2011;53(4):1405-6. author reply 1407. doi:10.1002/hep.24185. [PubMed: 21480360].

20. Heidrich B, Cordes HJ, Klinker H, Moller B, Naumann U, Rossle M, et al. Treatment Extension of Pegylated Interferon Alpha and Ribavirin Does Not Improve SVR in Patients with Genotypes 2/3 without Rapid Virological Response(OPTEX Trial): A Prospective, Randomized, Two-Arm, Multicentre Phase IV Clinical Trial. PLoS One. 2015;10(6). e0128069. doi: 10.1371/journal.pone.0128069. [PubMed: 26057627]. [PubMed Central: PMC4461366]. 